\title{
Materials for Optical Cryocoolers
}

\begin{abstract}
Markus P. Hehlen, ${ }^{* a}$ Mansoor Sheik-Bahae, ${ }^{b}$ Richard I. Epstein, ${ }^{\text {be }}$ Seth D. Melgaard ${ }^{\text {bc }}$ and Denis V. Seletskiy ${ }^{d}$

Vibration free cooling of detectors to cryogenic temperatures is critical for many terrestrial, airborne, and space based instruments. Cooling of solids by anti Stokes fluorescence is an emerging refrigeration technology that is inherently vibration free and compact, and enables cooling of small loads to cryogenic temperatures. In this Highlight, advances in laser cooling of solids are discussed with a particular focus on the recent breakthrough laser cooling of $\mathrm{Yb}^{3+}$ doped $\mathrm{YLiF}_{4}$ crystals to $114 \mathrm{~K}$. The importance of the material structure, composition, and purity of laser cooling materials and their influence on the optical refrigerator device performance is emphasized.
\end{abstract}

\section{Introduction}

Semiconductors find widespread use as sensors for both infrared (IR) light and ionizing radiation in many important applications. For example, $\mathrm{HgCdTe}$ and InSb are commonly used for the detection of long- $(8-12 \mu \mathrm{m})$ and mid-wave $(3-5 \mu \mathrm{m})$

${ }^{a}$ Materials Science \& Technology Division, Mailstop E549, Los Alamos National Laboratory, Los Alamos, NM 87545, USA. E mail: hehlen@lanl.gov; Tel: +1 505665 1737

${ }^{b}$ University of New Mexico, Physics and Astronomy Dept., 800 Yale Blvd. NE, Albuquerque, New Mexico 87131, USA

${ }^{c}$ Air Force Research Laboratory, Kirtland AFB, New Mexico 87117, USA

${ }^{d}$ Department of Physics and Center for Applied Photonics, University of Konstanz, D 78457 Konstanz, Germany

${ }^{e}$ ThermoDynamic Films LLC, Santa Fe, NM 87505, USA
IR light, ${ }^{1}$ respectively, and high-purity germanium (HPGe) is used for high-resolution gamma-ray spectroscopy. ${ }^{2}$ These materials are characterized by small bandgaps, $E_{\mathrm{g}}(0.15 \mathrm{eV}$ for $\mathrm{HgCdTe}, 0.23 \mathrm{eV}$ for InSb, and $0.73 \mathrm{eV}$ for Ge), making thermal excitation of an electron from the valence band to the conduction band a likely process at ambient temperatures. The respective dark current creates electrical (thermal) noise that degrades the performance of the detector. The magnitude of the thermal noise is proportional to $\exp \left(E_{\mathrm{g}} / 2 k T\right)$, and it can be sufficiently reduced by cooling the detector element to cryogenic temperatures $(<120 \mathrm{~K}) .{ }^{3}$ Cryogenic refrigeration is particularly challenging for many of the important portable, airborne, and space-based detector systems. Liquid cryogens are usually impractical on long-duration and weight-constrained missions, and thermo-electric (TEC) coolers are unsuited for many

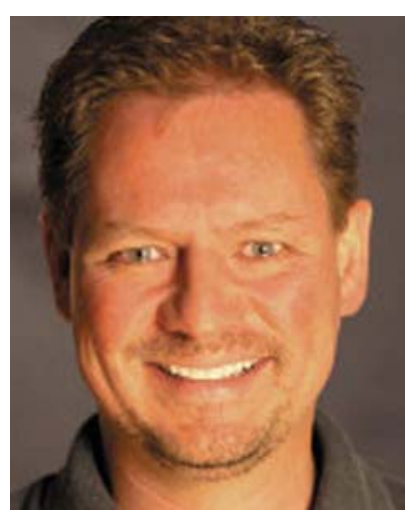

Markus P. Hehlen is a Staff Scientist at Los Alamos National Laboratory and Adjunct Research Associate Professor at the University of New Mexico. He received his Ph.D. in Inorganic Chemistry from the University of Bern, Switzerland, and conducted postdoctoral work at LANL and the University of Michigan. He was Senior Research Scientist and Project Manager at Gemfire Corporation, where he developed phosphors and fiber-optic amplifiers. He rejoined LANL in 2003 and focuses on the development of new optical materials and optoelectronic devices for defense, homeland security, and threat reduction applications. He is a Founding Associate Editor of Optical Materials Express.

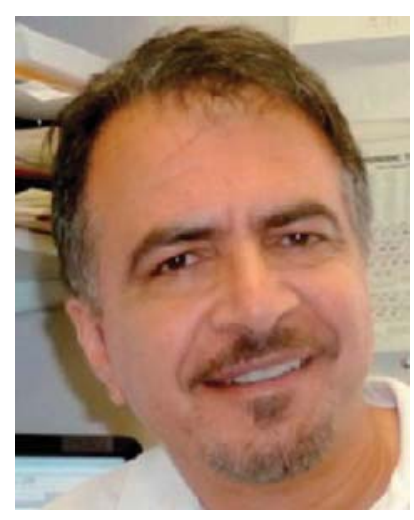

Mansoor Sheik-Bahae is a professor of Physics and Astronomy and the chair of Optical Science and Engineering at the University of New Mexico, Albuquerque, NM (USA). He graduated from the State University of New York (Buffalo), and subsequently spent seven years as a research scientist at CREOL - University of Central Florida before joining UNM in 1994, where he heads the Consortium for Laser Cooling of Solids. He has authored more than 200 scientific papers in nonlinear optics, ultrafast phenomena, and solid-state laser cooling, with more than 7000 citations to his work. He is a fellow of Optical Society of America and the recipient of the society's $R$. W. Wood prize in 2012. 
applications because they are limited to temperatures of $\sim 175 \mathrm{~K}$. This currently leaves mechanical coolers (e.g. Stirling, pulse tube, or reverse Brayton refrigerators) as the only available cryogenic technologies. Mechanical coolers however impart microphonic noise to the detector. Even in liquid-cryogencooled devices, microphonics can arise from the bubbling of the cryogen. In gamma-ray spectrometers, these vibrations cause capacitive changes in the electrical connections between the HPGe and the pre-amplifier that degrade the signal resolution, ${ }^{4}$ and in optical cameras they cause the image to blur. ${ }^{5}$ Despite significant progress in damping compressor vibrations ${ }^{5}$ and in digitally filtering detector signals, ${ }^{6}$ microphonics remains the major factor limiting detector resolution. This has fueled research into cryogenic cooling technologies that are free of vibrations.

Solid-state optical refrigeration is one of the most promising vibration-free cooling technologies. It is based on the process of anti-Stokes fluorescence (Fig. 1) in which a material emits light at wavelength $\tilde{\lambda}_{\mathrm{f}}$ after being excited by a laser at slightly longer

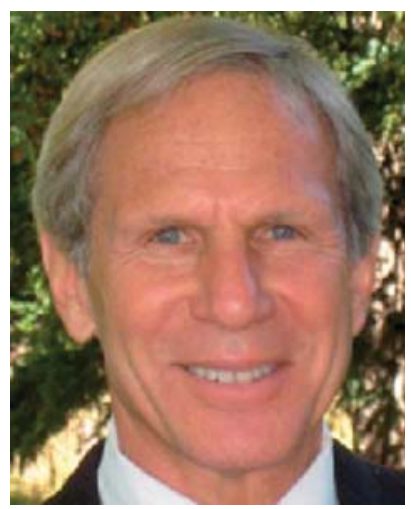

Richard I. Epstein is the CEO of ThermoDynamic Films LLC in New Mexico and an adjunct professor at the University of New Mexico. He was an undergraduate in Engineering Physics at Cornell University and received his Ph.D. in Applied Physics from Stanford University. He did research at the University of Texas at Austin, Harvard University and Nordita in Copenhagen, and then joined Los Alamos National Laboratory where he was a Laboratory Fellow and led the effort in optical refrigeration. He has published over 160 papers in theoretical astrophysics, satellite instrumentation, and applied physics. He is a fellow of the Optical Society of America.

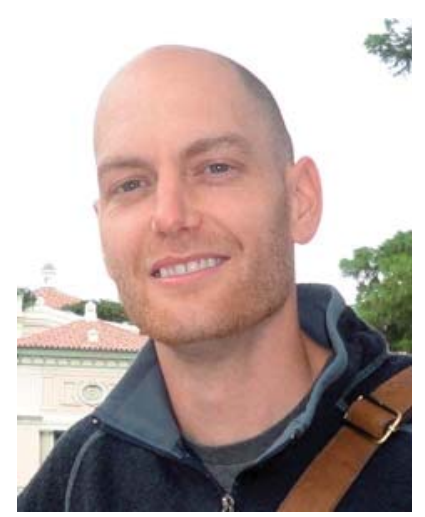

Seth D. Melgaard is a National Research Council Postdoctoral Fellow at the Air Force Research Labs in New Mexico. He received his Ph.D. in Optical Science and Engineering from the University of New Mexico in 2013. His multidisciplinary research interests include measurement and improvement of cryogenic optical refrigeration materials and components to create an all solid-state cryo-cooler for spacebased applications through trace element analysis, cryogenic payload cooling, thermal management, and optical trapping in resonant and non-resonant cavities.

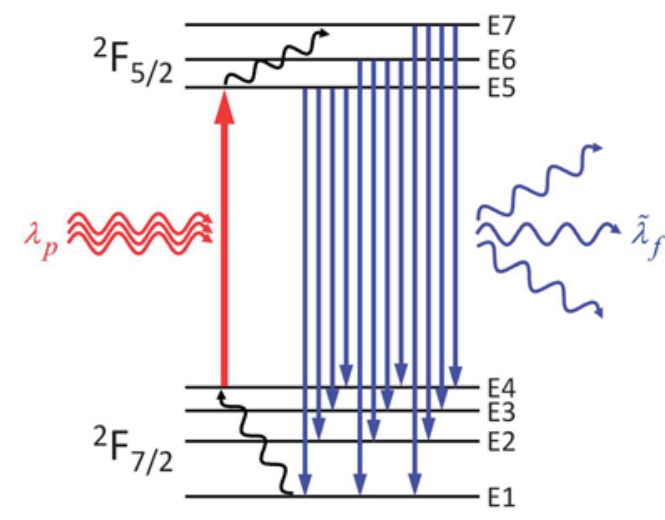

Fig. 1 Energy level diagram of $\mathrm{Yb}^{3+}$ illustrating removal of thermal energy from and thus cooling of a solid by anti Stokes fluorescence (average emission wave length shorter than the pump laser wavelength).

wavelength $\lambda_{\mathrm{p}}$. The respective energy difference is made up by the absorption of thermal energy from the material, thus cooling the solid. This cooling cycle is an all-optical process and is therefore inherently free of mechanical vibrations. Solid-state optical refrigeration has made tremendous progress since its first experimental demonstration by Epstein et al. in $1995 .^{7} \mathrm{~A}$ recent demonstration of a dramatic enhancement of the cooling efficiency in an ytterbium-doped fluoride crystal $\left(\mathrm{YLiF}_{4}: \mathrm{Yb}^{3+}\right)^{8}$ by Sheik-Bahae's group led to cooling of this material to $114 \mathrm{~K}$ starting from room temperature. ${ }^{9}$ This advance has put solidstate optical refrigeration within the cryogenic temperature range (defined as $<123 \mathrm{~K}$ by the National Institute of Standards and Technology, NIST). In this Highlight article, we review the latest results in this field and show that solid-state optical refrigeration is poised to enable the next generation of cryogenic refrigerators.

\section{Optical refrigeration of solids}

Consider a transparent solid doped with activator ions that have an electronic ground state and electronic excited state, each

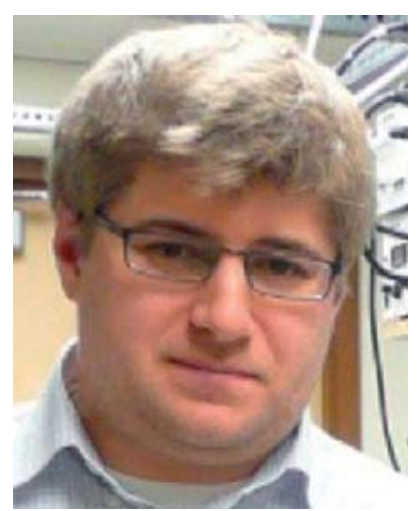

Denis V. Seletskiy is a National Science Foundation and Marie Curie Research Fellow at the University of Konstanz, Germany. He received his Ph.D. with distinction in Optical Science and Engineering from the University of New Mexico in 2010. His current research interests include solid-state laser cooling, nonlinear optics, and ultrafast phenomena. 
split into a number of sub-levels by the crystal field (see Fig. 1). Excitation of this system at energy $E_{\mathrm{p}}=h c / \lambda_{\mathrm{p}}$ is first followed by thermalization of the population among the crystal-field levels of the excited state. This process occurs via electron-phonon interactions on a picosecond time scale, and it establishes a Boltzmann equilibrium population in the excited state manifold among the ensemble of activator ions that is determined by the crystal-field splitting and the sample temperature. The excited state can subsequently decay by radiative relaxation, and the resulting luminescence spectrum consists of the numerous crystal-field transitions between the excited and the groundstate multiplets. The mean energy of the emitted photons, $\tilde{E}_{\mathrm{f}}=h c / \tilde{\lambda}_{\mathrm{f}}$, depends on the system temperature as well as the crystal-field splitting and oscillator strengths in the given material. If the system is excited at $E_{\mathrm{p}}<\tilde{E}_{\mathrm{f}}$ (regime of anti-Stokes fluorescence), then the energy difference $\Delta E=\tilde{E}_{\mathrm{f}} \quad E_{\mathrm{p}}$ required to establish thermal equilibrium has to be supplied by the thermally excited phonon bath of the solid. For each excitation/ emission cycle, thermal energy in the amount of $\Delta E$ is extracted from the solid and carried away as light, thus cooling the solid in the process. This cooling cycle was first proposed by Pringsheim in $1929,{ }^{10}$ and later work by Landau showed that it does not violate the second law of thermodynamics if the entropy of the light is considered as well. ${ }^{11}$ The cooling process converts low entropy laser light into higher entropy fluorescence in a cycle that is essentially a laser running in reverse. The cooling efficiency of this ideal case is given by $\eta_{\mathrm{c}}=\Delta E / E_{\mathrm{p}}=\left(\begin{array}{ll}\lambda_{\mathrm{p}} / \tilde{\lambda}_{\mathrm{f}} & \end{array}\right.$ Numerous parasitic processes can degrade this ideal cooling efficiency. They include non-radiative decay of the activator excited state, energy transfer to impurities that decay nonradiatively, and direct excitation of impurities that decay nonradiatively. One of the key objectives in creating a laser-cooling material is to minimize all non-radiative processes that cause parasitic heating.

Rare-earth ions have proven to be particularly suited as activator ions for solid-state laser cooling. Excitation and luminescence in rare-earth ions is primarily due to transitions between the electronic states of the respective $4 \mathrm{f}$ configuration. ${ }^{12}$ The $4 \mathrm{f}$ electrons are shielded quite effectively from the effects of the surrounding charges in a solid by the outer $5 \mathrm{~s}$ and $5 \mathrm{p}$ orbitals, which reduces electron-phonon coupling and leads to spectrally narrow transitions and high quantum efficiency for many of the $4 \mathrm{f}$ excited states. The $\mathrm{Yb}^{3+}$ ion has gained particular attention for laser cooling because, unlike most other rare-earth ions, it has only one excited state, ${ }^{2} \mathrm{~F}_{5 / 2}$, that is located $\sim 1.2 \mathrm{eV}$ above the ground state, ${ }^{2} \mathrm{~F}_{7 / 2}$ (see Fig. 1). Transitions between ${ }^{2} \mathrm{~F}_{7 / 2}$ and ${ }^{2} \mathrm{~F}_{5 / 2}$ occur at wavelengths of $\sim 1 \mu \mathrm{m}$ where powerful and reliable solidstate lasers are available. The absence of any other excited states eliminates many processes such as upconversion, cross relaxation, and radiative relaxation between excited states, which would lead to heating. Of all rare-earth ions, $\mathrm{Yb}^{3+}$ most closely resembles the ideal case depicted in Fig. 1. It is therefore not surprising that most of the work in solid-state optical refrigeration has been performed on $\mathrm{Yb}^{3+}$-doped solids, ${ }^{13}$ beginning with the first observation of laser cooling in the $\mathrm{Yb}^{3+}$-doped fluoride glass $\mathrm{ZBLAN}^{7}$ and culminating in the recent laser cooling to $114 \mathrm{~K}$ in the $\mathrm{Yb}^{3+}$-doped fluoride crystal $\mathrm{YLiF}_{4} \cdot{ }^{9}$
In parallel to advances in laser cooling in rare-earth doped materials, optical refrigeration in semiconductors has been extensively investigated. ${ }^{\mathbf{1 4} 18}$ Net cooling in bulk semiconductors (GaAs heterostructures) has been elusive primarily due to a large parasitic background absorption. In a surprising development, however, Xiong's group at Nanyang Technological University (Singapore) recently reported laser cooling of bare CdS nanostructures (nanobelts) by $40 \mathrm{~K} .{ }^{19}$ Further investigation into understanding and advancing this work towards cryogenic temperatures and scaling to larger structures promises to be highly rewarding.

\section{Design and fabrication of laser-cooling materials}

It is instructional to take a closer and more formal look at the various factors that determine the laser-cooling efficiency and thus the ultimate temperature that can be achieved by a solidstate optical refrigerator. Sheik-Bahae and Epstein performed a rate-equation analysis of the energy-level scheme shown in Fig. 1 and found that the laser cooling efficiency is given by ${ }^{\mathbf{1 3 2 0}}$

$$
\eta_{\mathrm{c}}\left(\lambda_{\mathrm{p}}, T\right)=\eta_{\mathrm{ext}} \times \eta_{\mathrm{abs}}\left(\lambda_{\mathrm{p}}, T\right) \times \frac{\lambda_{\mathrm{p}}}{\tilde{\lambda}_{\mathrm{f}}(T)} \quad 1
$$

where $\eta_{\mathrm{c}}>0$ indicates net cooling and $\eta_{\mathrm{c}}<0$ indicates net heating of the system. In eqn (1), $\eta_{\text {ext }}$ is the external quantum efficiency $\eta_{\text {ext }}=\eta_{\mathrm{e}} W_{\mathrm{r}} /\left(\eta_{\mathrm{e}} W_{\mathrm{r}}+W_{\mathrm{nr}}\right)$, where $\eta_{\mathrm{e}}$ is the efficiency with which the fluorescence can be extracted from the sample, and $W_{\mathrm{r}}$ and $W_{\mathrm{nr}}$ are the radiative and non-radiative decay rates of the excited state, respectively. Furthermore in eqn (1), the absorption efficiency is a measure of how much of the total absorbed power at $\lambda_{\mathrm{p}}$ is absorbed by $\mathrm{Yb}^{3+}$ (rather than by impurities) i.e.

$$
\eta_{\text {abs }}\left(\lambda_{\mathrm{p}}, T\right)=\frac{\alpha_{\mathrm{r}}\left(\lambda_{\mathrm{p}}, T\right)}{\alpha_{r}\left(\lambda_{\mathrm{p}}, T\right)+\alpha_{\mathrm{b}}\left(\lambda_{\mathrm{p}}\right)} .
$$

Here, $\alpha_{\mathrm{r}}$ is the "resonant" absorption coefficient of $\mathrm{Yb}^{3+}$ for the transition that starts the cooling cycle, and $\alpha_{\mathrm{b}}$ is the background absorption coefficient induced by impurities or defects present in the material. Effects of saturation have been ignored. ${ }^{13}$ In this model, impurities can induce non-radiative quenching of the $\mathrm{Yb}^{3+}$ excited state $\left(W_{\mathrm{nr}}>0\right)$ and cause direct absorption of pump light $\left(\alpha_{\mathrm{b}}>0\right)$. Both of these processes produce parasitic internal heating and thus degrade the cooling efficiency.

As the temperature of the cooling element decreases to cryogenic temperatures, additional cooling becomes increasingly challenging for two reasons. ${ }^{13}$ First, the mean fluorescence wavelength $\tilde{\lambda}_{\mathrm{f}}$ red-shifts with decreasing temperature because higher-energy crystal-field levels in the excited state become depopulated (see Fig. 1). As a result, the corresponding ratio $\lambda_{\mathrm{p}} / \tilde{\lambda}_{\mathrm{f}}(T)$ in eqn (1) approaches unity, thus decreasing $\eta_{\mathrm{c}}$. Second, the resonant absorption coefficient $\alpha_{\mathrm{r}}$ at $\lambda_{\mathrm{p}}$ decreases exponentially with decreasing temperature because the upper crystal-field level in the ground state becomes depopulated. In contrast, for an ytterbium-based optical refrigerator, absorption transitions of important impurities (3d transition metal ions 
such as $\mathrm{Fe}^{2+}, \mathrm{Cu}^{2+}, \mathrm{Co}^{2+}$, etc.) do not change significantly at low temperatures, leading to an $\alpha_{\mathrm{b}}$ that remains relatively constant with temperature. This causes $\eta_{\mathrm{abs}}\left(\lambda_{\mathrm{p}}, T\right)$ to decrease dramatically as the sample cools. Combined, these two effects set a lower limit - also known as the minimum achievable temperature $(\mathrm{MAT})^{\mathbf{1 3}}$ - for the laser-cooling temperature for a given material.

The above considerations highlight the materials chemistry and optical engineering challenges that have to be overcome in order to realize a cryogenic solid-state laser refrigerator. The challenges fall in three groups:

(1) Choice of host material: the excited state of $\mathrm{Yb}^{3+}$ can decay non-radiatively by transferring its energy to vibrational modes of the host material. The probability of this multi-phonon relaxation decreases exponentially with the number of phonons that are created in the process. The host material therefore has to have a sufficiently low phonon energy such that multiphonon relaxation rate of the $\mathrm{Yb}^{3+}$ excited state $\left(W_{\mathrm{nr}}\right)$ is much lower than the radiative relaxation rate $\left(W_{\mathrm{r}}\right)$, i.e. $\eta_{\mathrm{int}} \rightarrow 1$. Hehlen has shown that for $\mathrm{Yb}^{3+}$ this condition is marginally met for some oxide crystals and is well met for all common halide materials, which have relatively low phonon energies in the 400-600 $\mathrm{cm}^{-1}$ range. ${ }^{21}$ Optical refrigeration has been observed in a variety of glasses and crystals with relatively low phonon energy, such as $\mathrm{Yb}^{3+}$-doped materials (ZBLANP, ${ }^{7,22}{ }^{25} \mathrm{ZBLAN}^{26}{ }^{21}$ ZBLANI, ${ }^{32} \mathrm{CNBZn},{ }^{33,34} \mathrm{BIG},{ }^{28,35,36} \mathrm{KGd}\left(\mathrm{WO}_{4}\right)_{2},{ }^{37} \mathrm{KY}\left(\mathrm{WO}_{4}\right)_{2},{ }^{37}$ $\mathrm{YAG}^{37,38} \mathrm{Y}_{2} \mathrm{SiO}_{5},{ }^{38} \quad \mathrm{KPb}_{2} \mathrm{Cl}_{5},{ }^{33,39} \mathrm{BaY}_{2} \mathrm{~F}_{8},{ }^{40,41} \mathrm{YLF},{ }^{9,42}$ 44, 53, 55 $\mathrm{ABCYS}^{45}$ ), $\mathrm{Tm}^{3+}$-doped materials (ZBLAN, ${ }^{46,47} \mathrm{BYF}^{48}$ ), and $\mathrm{Er}^{3+}$ doped materials (CNBZn, ${ }^{49} \mathrm{KPb}_{2} \mathrm{Cl}_{5}$ (ref. 49 and 50)). Multiphonon relaxation of the $\mathrm{Yb}^{3+}$ excited state is negligible in fluoride crystals and glasses. Furthermore, fluorides are particularly attractive because they tend to be chemically inert, which greatly facilitates material preparation and handling as well as improves long-term material reliability.

(2) Material purity: laser light absorbed by impurities does not generally lead to anti-Stokes fluorescence, but rather is converted to heat. This requires the material to be free of impurities $\left(\alpha_{\mathrm{b}} \rightarrow 0\right)$. Of particular concern are impurities that have optical absorptions around $1 \mu \mathrm{m}$ ( $\mathrm{Yb}^{3+}$ absorption) such as $3 \mathrm{~d}$ transition metal ions. These ions get excited along with $\mathrm{Yb}^{3+}$ and cause internal heating which becomes particularly pronounced at cryogenic temperatures where $\eta_{\mathrm{abs}}\left(\lambda_{\mathrm{p}}, T\right)$ is small (eqn (2)). Hehlen et al. have recently shown that material purities of at least $99.99999 \%$ (i.e., $100 \mathrm{ppb}$ ) with respect to transition-metal ions are required to achieve laser cooling to cryogenic temperatures. ${ }^{51}$ Commercial-grade starting materials do not have sufficient purity, and additional purification by solvent extraction, zone refining, or recrystallization is required for this application. ${ }^{52}$

(3) Resonant absorption: the absorption efficiency $\eta_{\text {abs }}$ can also be increased by increasing the resonant absorption, $\alpha_{\mathrm{r}}$, by $\mathrm{Yb}^{3+}$. This can be accomplished by increasing the $\mathrm{Yb}^{3+}$ doping level. Unfortunately, high doping levels often increase energy migration among the $\mathrm{Yb}^{3+}$ ions which leads to non-radiative quenching at impurity sites. ${ }^{51}$ Alternatively, the absorbed power can be increased by increasing the laser path length in the sample, for example by placing the material in a non-resonant cavity in order to create a multi-pass pump geometry (see Fig. 2). Finally, a large improvement in $\alpha_{\mathrm{r}}$ can be achieved by using crystalline materials instead of glasses. The amorphous structure of glasses creates a distribution of $\mathrm{Yb}^{3+}$ chemical coordination geometries and respective crystal fields, causing an inhomogeneous broadening of the optical transitions. In contrast, $\mathrm{Yb}^{3+}$ ions are incorporated on one preferred crystallographic site in many crystals and therefore all experience the same crystal field, giving rise to spectrally narrow transitions. ${ }^{8}$ The significantly smaller inhomogeneous broadening in crystals compared to glasses results in a correspondingly higher peak absorption cross section at $\lambda_{\mathrm{p}}$ for the same level of $\mathrm{Yb}^{3+}$ doping. This last point has been studied in detail by Seletskiy et al. who have analyzed ${ }^{54}$ and experimentally verified ${ }^{55}$ the laser cooling efficiency model for ZBLAN: $\mathrm{Yb}^{3+}$ glass and $\mathrm{YLiF}_{4}: \mathrm{Yb}^{3+}$ (YLF:Yb) crystal (see Fig. 3). Even though $\eta_{\text {ext }}$ and $\alpha_{\mathrm{b}}$ are similar for both samples studied, the minimum achievable temperature in the YLF: $\mathrm{Yb}$ crystal is much lower $(110 \mathrm{~K})$ than in the ZBLAN: $\mathrm{Yb}^{3+}$ glass $(190 \mathrm{~K})$. This is a direct result of the higher peak absorption cross section in the crystal compared to the glass. The MAT of $110 \mathrm{~K}$ in YLF:Yb is obtained when the pump laser is tuned to the E4 $\rightarrow$ E5 crystal-field absorption (see Fig. 1). Research on solid-state optical refrigeration was originally performed on $\mathrm{Yb}^{3+}$-doped fluoride glasses (such as ZBLAN) because high-purity samples of these materials were commercially available in the late 1990s from the fiber-optic telecommunications industry. As illustrated in Fig. 4, steady progress was achieved in the early years with ZBLAN: $\mathrm{Yb}^{3+}$ as many of the materials and optical engineering aspects were developed and the material approached its intrinsic laser-cooling limit. The importance of small inhomogeneous broadening for cryogenic operation, however, is a more recent insight. ${ }^{8}$ This insight, first implemented in fluoride crystals by Bigotta et al. in $2007,{ }^{43}$ has ushered in a new chapter in solid-state optical refrigeration, paving the way to cryogenic temperatures.

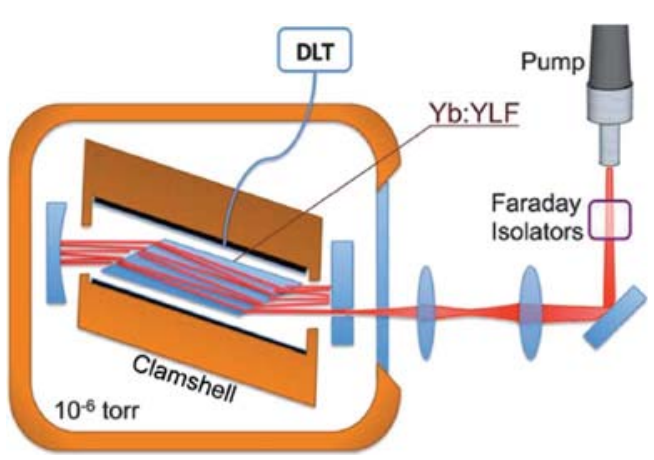

Fig. 2 Schematic of the experimental setup used by Melgaard et al. to achieve laser cooling of YLF:Yb crystals to cryogenic temperatures.9,53 The pump laser makes multiple passes through the YLF:Yb crystal (center, blue), which is enclosed in a tightly fitting copper "clamshell" coated with spectrally selective Maxorb ${ }^{\text {TM }}$ foil (black layers). The components are enclosed in a chamber under vacuum. Also shown is an optical fiber used to collect some of the YLF:Yb fluorescence for the purpose of non contact differential luminescence thermometry (DLT). Reprinted from S. D. Melgaard, D. V. Seletskiy, A. Di Lieto, M. Tonelli, and M. Sheik Bahae, "Optical refrigeration progress: cooling below NIST cryogenic temperature of 123 K", Proc. SPIE, 2013, 8638, 863804, with permission from SPIE. 

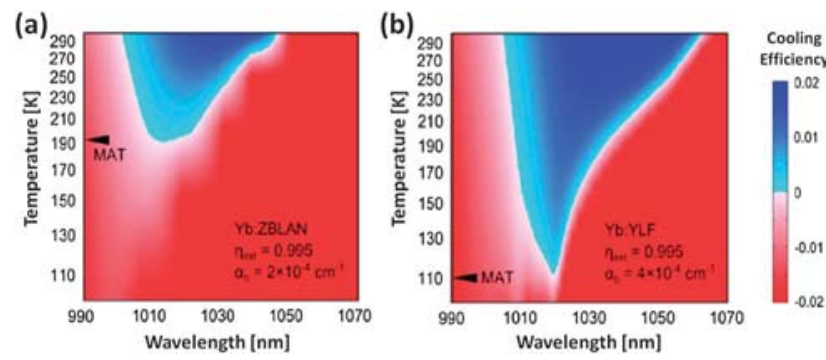

Fig. 3 Calculated maps of cooling efficiency as a function of pump lase wavelength for (a) a $\mathrm{Yb}^{3+}$ doped ZBLAN glass and (b) a $\mathrm{Yb}^{3+}$ doped YLF crystal. ${ }^{54}$ Red regions denote laser induced heating $\left(\eta_{c}<0\right)$ while blue regions denote laser induced cooling $\left(\eta_{\mathrm{c}}>0\right)$. The minimum achievable temperature is indicated as MAT for both samples. Reprinted from J. Lumin., 133, D. V. Seletskiy, S. D. Melgaard, R. I. Epstein, A. Di Lieto, M. Tonelli, and M. Sheik Bahae, "Precise determination of minimum achievable temperature for solid state optical refrig eration", pp. 5 9, Copyright (2013), with permission from Elsevier.

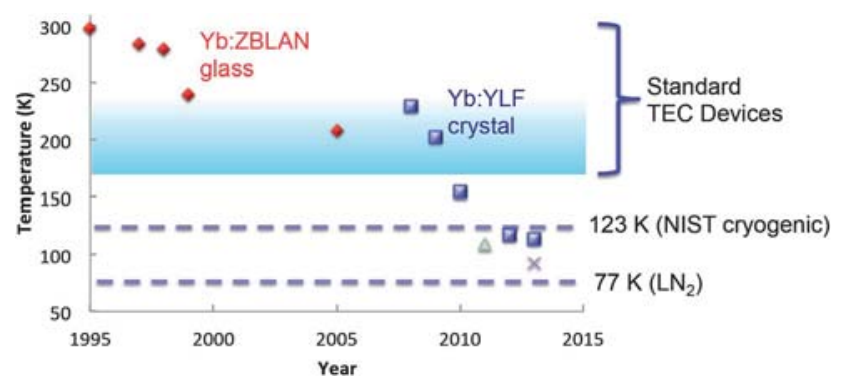

Fig. 4 Progress towards cryogenic solid state laser cooling since the first experimental observation by Epstein et al. in 1995. Devices built using YLF:Yb crystals have recently broken the cryogenic barrier and are approaching liquid nitrogen temperatures. The diamonds and squares are experimental results while the triangle and cross are calculated minimum achievable temperatures for some YLF:Yb crystals.

\section{Cryogenic laser-cooling of $\mathrm{Yb}^{3+}$-doped $\mathrm{YLiF}_{4}$ crystals}

The research group of Sheik-Bahae at the University of New Mexico has been the first to laser-cool a solid to the NISTdefined cryogenic regime from room temperature., ${ }^{9,53}$ They used $5 \mathrm{~mol} \%$ and $10 \mathrm{~mol} \% \mathrm{Yb}^{3+}$-doped YLF single crystals that were grown by AC Materials Inc. using the Czochralski method. The preparation started from ultra-pure $\mathrm{YF}_{3}, \mathrm{LiF}$, and $\mathrm{YbF}_{3}$ precursors to minimize transition-metal impurities, and it also involved pretreatment of the precursors in hot HF gas to reduce oxygen impurities. The crystals had a length of $12 \mathrm{~mm}$ and Brewster faces cut for a parallel orientation of the laser polarization $(\vec{E})$ with respect to the crystal unique axis $(\vec{c})$. This crystal orientation was chosen to maximize the absorption cross section of the E4 $\rightarrow$ E5 crystal-field transition (see Fig. 1). Characterization of the $5 \mathrm{~mol} \% \mathrm{Yb}^{3+}$-doped crystal confirmed a high external quantum efficiency of $\eta_{\text {ext }}=99.5 \%$ for the $\mathrm{Yb}^{3+}$ ${ }^{2} \mathrm{~F}_{5 / 2} \rightarrow{ }^{2} \mathrm{~F}_{7 / 2}$ emission and a low background absorption coefficient of $\alpha_{\mathrm{b}}=4.6 \times 10^{-4} \mathrm{~cm}^{-1}$ at the $1020 \mathrm{~nm}$ pump laser wavelength. ${ }^{9}$ The $10 \mathrm{~mol} \% \mathrm{Yb}^{3+}$-doped sample had even better parameters $\left(\eta_{\text {ext }}=99.6 \% \text { and } \alpha_{\mathrm{b}}=2.0 \times 10^{-4} \mathrm{~cm}^{-1}\right)^{9}$ that indicated lower parasitic heating while offering twice the resonant absorption cross section compared to the $5 \mathrm{~mol} \%$ crystal. This is reflected in the minimum achievable temperatures (MAT) that were calculated to $118 \mathrm{~K}$ and $93 \mathrm{~K}$ for the $5 \mathrm{~mol} \%$ and $10 \mathrm{~mol} \%$ doped crystals, respectively, based on these parameters. $^{9}$

The experimental setup is shown in Fig. 2, and it was developed with the goal to optimize both optical and thermal device performance. The YLF:Yb crystal was placed in a Herriott cell, ${ }^{56}$ which was formed by a highly reflective flat input coupler mirror and a curved $(R=25 \mathrm{~cm})$ back reflector separated by $35 \mathrm{~mm} .{ }^{9,53}$ The pump laser beam entered the non-resonant cavity though a 1-mm diameter hole in the input coupler and performed 5 round-trip passes through the crystal before unabsorbed pump light exited the input aperture to be dumped externally. A linearly-polarized single-mode ytterbium fiber laser (IPG Photonics) operating at $1020 \mathrm{~nm}$ with a maximum power of $50 \mathrm{~W}$ (continuous wave) was used to excite the E4 $\rightarrow$ E5 transition of $\mathrm{Yb}^{3+}$ in YLF (see Fig. 1), which has to be pumped to approach the minimum achievable temperature (see Fig. $3 \mathrm{~b}$ ). The pump light can be supplied through free space or an optical fiber, in either case effectively isolating the optical refrigerator from any vibrations that may be present in the remote pump laser unit.

When the device is in thermal equilibrium at the lowest temperature, the laser-cooling power provided by the YLF:Yb crystal matches the thermal load from the environment on the YLF:Yb crystal. For the device to approach its MAT it is therefore essential to design a cooling chamber that minimizes the heat load on the laser-cooling crystal which contains convective, conductive, and radiative contributions. The convective heat transport is effectively eliminated by creating a vacuum $\left(10^{-6}\right.$ torr $)$ inside the cooling chamber. The residual thermal transport by the air in the chamber was estimated to be $\sim 10^{-7} \mathrm{~W} \mathrm{~K}^{-1} \cdot{ }^{57}$ The heat leakage by air was small compared to the conductive heat load, which is a result of heat flowing from the clamshell through the mechanical support structure into the crystal. This contribution can be reduced by minimizing the mechanical contact area between the crystal and the support structure by mounting the crystal with six optical fibers $(500 \mu \mathrm{m}$ diameter; thermal conductivity $\sim 1.0 \mathrm{~W} \mathrm{~m}^{-1} \mathrm{~K}$ ) protruding from the chamber walls. A residual thermal conductance of $\sim 10^{-5} \mathrm{~W} \mathrm{~K}^{-1}$ was estimated for this design..$^{57}$ By far the largest contribution to the total heat load on the laser-cooling crystal, however, was found to be radiative heat transport given by: $:^{47,57,58}$

$$
P_{\mathrm{rad}}=\frac{\varepsilon_{\mathrm{s}} \sigma A_{\mathrm{s}}\left(\mathrm{T}_{\mathrm{c}}{ }^{4} \mathrm{~T}_{\mathrm{s}}{ }^{4}\right)}{1+\chi},
$$

where the subscripts $\mathrm{s}$ and $\mathrm{c}$ denote the sample and chamber, respectively, $\chi=\left(\begin{array}{ll}1 & \varepsilon_{\mathrm{c}}\end{array}\right) \varepsilon_{\mathrm{s}} A_{\mathrm{s}} / \varepsilon_{\mathrm{c}} A_{\mathrm{c}}, \varepsilon$ is the thermal emissivity, $A$ is the area, and $\sigma$ is the Stefan-Boltzmann constant. Inspection of eqn (3) reveals that the radiative heat load can be minimized by tightly fitting the clamshell to the shape of the YLF:Yb crystal such that $A_{\mathrm{c}} \approx A_{\mathrm{s}}$ and by minimizing the thermal emissivity of the chamber walls such that $\varepsilon_{\mathrm{c}}<\varepsilon_{\mathrm{s}}$. The latter can be achieved by coating the inside surfaces with a spectrally-selective material that readily absorbs the fluorescent radiation but has low 
emissivity at black-body wavelengths (see Fig. 2). ${ }^{25}$ For example, Maxorb $^{\mathrm{TM}}$ is a treated nickel surface having a high solar-spectrum absorptivity of 0.97 and a low infrared emissivity of $\varepsilon_{\mathrm{c}}=0.08,{ }^{59,60}$ enabling efficient absorption of the YLF:Yb fluorescence while minimizing $P_{\text {rad }}$. From experiments on the warming dynamics of the optical refrigerator, ${ }^{58}$ a radiative heat load at the lowest temperatures of $\sim 2.4 \times 10^{-4} \mathrm{~W} \mathrm{~K}^{-1}$ $\left(\sim 1.4 \times 10^{-3} \mathrm{~W} \mathrm{~K}^{-1}\right.$ without the Maxorb ${ }^{\mathrm{TM}}$ coated clamshell $)$ was estimated, ${ }^{57}$ highlighting the dominance of radiative contribution to the heat load. A further reduction of $P_{\text {rad }}$ could be achieved by reducing the clamshell temperature $T_{\mathrm{c}},{ }^{53}$ for example with an external cooling stage such as a vibration-free TEC.

The coefficient of performance (COP) for a solid-state optical refrigerator is given by the ratio of cooling power and supplied wall-plug power, i.e. the product of the cooling efficiency, the laser coupling efficiency (the fraction of incident laser power absorbed by the crystal), and the pump laser wall-plug efficiency. The cooling efficiency is temperature dependent (see Fig. 3) and decreases from 2-5\% at room temperature (typical for $\mathrm{Yb}^{3+}$ doped crystals) to zero at the MAT. The coupling efficiency can approach $100 \%$ if the crystal is placed in a cavity geometry (see Fig. 2). Advanced semiconductor diode lasers can reach wall plug efficiencies as high as $75 \%$. The COP of a solidstate optical refrigerator will therefore vary from $1.5-3.5 \%$ at room temperature to zero at the MAT in the cryogenic regime. However, if the "waste" fluorescence from the sample is harvested and recycled by means of (for example) photo-voltaic convertors, it has been shown that the overall COP will only be limited by the Carnot efficiency. ${ }^{61}$

The cooling performance of a laser refrigerator using a $10 \mathrm{~mol} \% \mathrm{Yb}^{3+}$-doped YLF crystal is shown in Fig. 5. The YLF:Yb crystal reached a steady-state temperature of $114 \mathrm{~K} \pm 1 \mathrm{~K}$ within 13 minutes using an incident 1020-nm laser power of $45 \mathrm{~W}$ (of which $20 \mathrm{~W}$ were absorbed at $114 \mathrm{~K}$ ). Concurrently, the clamshell temperature increased to $299 \mathrm{~K}$ as a result of the YLF:Yb fluorescence absorbed by the chamber walls. The heat lift by the YLF:Yb crystal at $114 \mathrm{~K}$ was estimated to $190 \mathrm{~mW}$, which is the current world-record in solid-state optical refrigeration. As

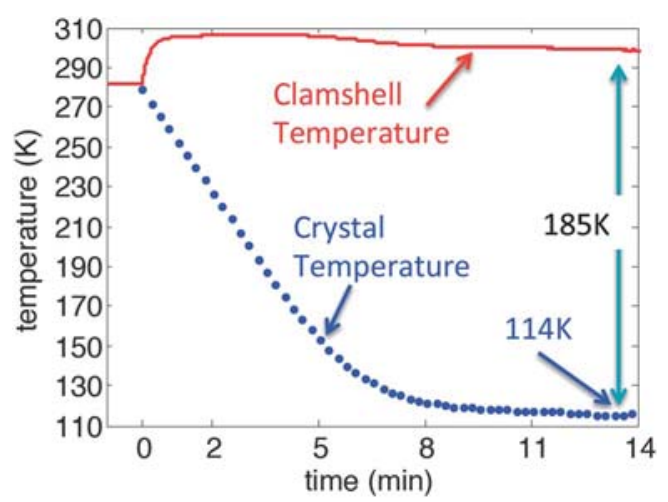

Fig. 5 Cooling of a $10 \mathrm{~mol} \% \mathrm{Yb}^{3+}$ doped YLF crystal. The $45 \mathrm{~W}$ pump laser operating at $1020 \mathrm{~nm}$ was turned on at $t=0$. Reprinted from S. D. Melgaard, D. V. Seletskiy, A. Di Lieto, M. Tonelli, and M. Sheik Bahae, "Optical refrigeration progress: cooling below NIST cryogenic temperature of 123 K", Proc. SPIE, 2013, $\mathbf{8 6 3 8}, 863804$, with permission from SPIE. expected from the larger $\alpha_{\mathrm{b}}$ and lower $\alpha_{\mathrm{r}}$, the $5 \mathrm{~mol} \% \mathrm{Yb}^{3+}$ doped YLF crystal showed worse performance by reaching a steady-state temperature of $123 \mathrm{~K}$. Using the $5 \mathrm{~mol} \% \mathrm{Yb}^{3+}$ doped crystal, Melgaard et al. used liquid nitrogen to cool the clamshell from room temperature to $208 \mathrm{~K}$, and they observed the YLF:Yb crystal temperature to decrease from $123 \mathrm{~K}$ to $119 \mathrm{~K}$ as a result of the reduced radiative load of the clamshell on the crystal (see eqn (3)). This lower temperature is within less than a degree from the predicted MAT of $118 \mathrm{~K}$ for the $5 \mathrm{~mol} \%$ crystal, in full quantitative agreement with the laser cooling model.

\section{Device design and material chemistry challenges}

Work is underway to use this laser-refrigerator for cooling a practical load such as a semiconductor detector. ${ }^{57}$ Simply attaching the detector to the laser-cooling crystal is impractical because it would absorb the multi-Watt $\mathrm{Yb}^{3+}$ fluorescence, rapidly heat up, and degrade the device performance. Rather, a thermal link is being developed that prevents $\mathrm{Yb}^{3+}$ fluorescence from reaching the payload while offering high thermal conductivity for the heat to flow from the payload through the thermal link to the YLF:Yb crystal where it is carried away as anti-Stokes fluorescence. A solid piece of sapphire having a right-angle kink is an attractive design choice. ${ }^{57}$ Sapphire offers high thermal conductivity, and the $90^{\circ}$ kink rejects most of the $\mathrm{Yb}^{3+}$ fluorescence entering the link. An additional metal coating at the link endface can then be used to block any residual fluorescence from reaching the attached payload. The design of the thermal link involves optimization of the optical geometry as well as careful choice of the methods and materials used to bond the various components.

Another promising area for advances is the purity of the laser-cooling crystal. Melgaard et al. have conducted a recent study of transition metal impurities in YLF:Yb crystals. ${ }^{57}$ They dissolved YLF:Yb material by boiling it in a mixture of $15 \mathrm{~N}$ $\mathrm{HNO}_{3}$ and $6 \mathrm{~N} \mathrm{HCl}$ in a Teflon vessel for several hours, followed by a workup of the solution and trace-element analysis using a inductively-coupled plasma (ICP) mass spectrometry. Various crystals were found to contain hundreds of ppb of impurities such as $\mathrm{Fe}, \mathrm{Co}, \mathrm{Ni}, \mathrm{Cu}, \mathrm{Cr}$, and $\mathrm{V}$, with the $\mathrm{Fe}$ impurity level being the largest. Fig. 6 shows that the background absorption coefficient at $1020 \mathrm{~nm}, \alpha_{\mathrm{b}}$, scales with the Fe impurity concentration, providing evidence that iron impurities play an important role in inducing parasitic heating. Applying this result to the laser-cooling model developed earlier (see Section 3) leads us to predict that a reduction in transition metal impurities by $10 \times$ from the current record-cooling samples should enable laser cooling to temperatures near $80 \mathrm{~K}$ (ref. 13) (see Fig. 6, right). Patterson et al. have shown earlier that metal fluorides such as $\mathrm{ZrF}_{4}, \mathrm{BaF}_{2}, \mathrm{LaF}_{3}, \mathrm{AlF}_{3}, \mathrm{NaF}, \mathrm{InF}_{3}$, and $\mathrm{YbF}_{3}$ having $<100 \mathrm{ppb}$ transition metal purities can be prepared using chelate-assisted solvent extraction. ${ }^{52}$ Applying these processes to the $\mathrm{YF}_{3}, \mathrm{LiF}$, and $\mathrm{YbF}_{3}$ precursors of $\mathrm{YLF}: \mathrm{Yb}$ followed by crystal growth under tightly controlled conditions may enable the next leap to sub-100 K temperatures. 

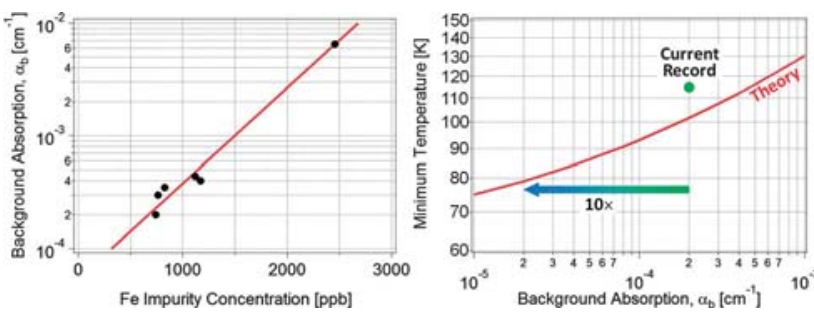

Fig. 6 (left) Correlation between the background absorption coefficient at $1020 \mathrm{~nm}, \alpha_{\mathrm{b}}$, and the iron impurity concentration; (right) dependence of the MAT on $\alpha_{\mathrm{b}}$, indicating that a $10 \times$ improvement in transition metal impurities is expected to enable laser cooling to near $80 \mathrm{~K}$.

\section{Summary and outlook}

Optical refrigeration of solids has come a long way since its theoretical inception by Pringsheim in 1929 and its first experimental observation as a modest $0.3 \mathrm{~K}$ temperature drop in ZBLAN:Yb glass by Epstein et al. in 1995. The latest optical refrigerators based on $\mathrm{Yb}^{3+}$-doped $\mathrm{YLiF}_{4}$ crystals developed by the Sheik-Bahae group at the University of New Mexico have now broken the cryogenic barrier. Laser cooling to $114 \mathrm{~K}$ with a heat lift of $190 \mathrm{~mW}$ has been demonstrated, bringing this refrigeration technology to the forefront for applications that require vibration-free cooling to cryogenic temperatures. Several advances in both materials chemistry and in cryocooler device design are still needed to reach temperatures near $80 \mathrm{~K}$.

\section{References}

1 A. Rogalski, Infrared Detectors, CRC Press, Boca Raton, 2011.

2 G. F. Knoll, Radiation Detection and Measurement, John Wiley \& Sons Inc., New York, 3rd ed, 2000.

3 A. Rogalski, Rep. Prog. Phys., 2005, 68, 2267-2336.

4 G. Gilmore and J. D. Hemingway, Practical Gamma-Ray Spectrometry, John Wiley \& Sons Inc., New York, 1998.

5 A. M. Veprik, V. I. Babitsky, N. Pubdak and S. V. Riabzev, Cryogenics, 2009, 49, 449-454.

6 M. K. Schultz, R. M. Keyser, R. C. Trammell and D. L. Upp, J. Radioanal. Nucl. Chem., 2007, 271, 101-106.

7 R. I. Epstein, M. I. Buchwald, B. C. Edwards, T. R. Gosnell and C. E. Mungan, Nature, 1995, 377, 500-503.

8 D. V. Seletskiy, S. D. Melgaard, S. Bigotta, A. Di Lieto, M. Tonelli and M. Sheik-Bahae, Nat. Photonics, 2010, 4, 161-164.

9 S. D. Melgaard, D. V. Seletskiy, A. Di Lieto, M. Tonelli and M. Sheik-Bahae, Proc. SPIE, 2013, 8638, 863804.

10 P. Pringsheim, Z. Phys., 1929, 57, 739-746.

11 L. Landau, J. Phys., 1946, 10, 503-506.

12 M. P. Hehlen, M. G. Brik and K. W. Krämer, J. Lumin., 2013, 136, 221-239.

13 D. V. Seletskiy, M. P. Hehlen, R. I. Epstein and M. SheikBahae, Adv. Opt. Photonics, 2012, 4, 79-107.

14 H. Gauck, T. H. Gfroerer, M. J. Renn, E. A. Cornell and K. A. Bertness, Appl. Phys. A, 1997, 64, 143.

15 M. Sheik-Bahae and R. I. Epstein, Phys. Rev. Lett., 2004, 92, 24740 .
16 M. Sheik-Bahae and R. I. Epstein, Nat. Photonics, 2007, 1, 693-699.

17 G. Rupper, N. H. Kwong and R. Binder, Phys. Rev. Lett., 2006, 97, 117401.

18 J. B. Khurgin, J. Appl. Phys., 2006, 100, 113116.

19 J. Zhang, D. Li, R. Chen and Q. Xiong, Nature, 2013, 493, 504-508.

20 M. Sheik-Bahae and R. I. Epstein, Laser Photonics Rev., 2009, 3, 67-84.

21 M. P. Hehlen, Design and fabrication of rare-earth-doped laser cooling materials, in Optical Refrigeration: Science and Applications of Laser Cooling of Solids, ed. R. I. Epstein and M. Sheik-Bahae, Wiley-VCH Verlag, Weinheim, 2009.

22 C. E. Mungan, M. I. Buchwald, B. C. Edwards, R. I. Epstein and T. R. Gosnell, Phys. Rev. Lett., 1997, 78, 1030.

23 X. Luo, M. D. Eisaman and T. R. Gosnell, Opt. Lett., 1998, 23, 639.

24 T. R. Gosnell, Opt. Lett., 1999, 24, 1041.

25 J. Thiede, J. Distel, S. R. Greenfield and R. I. Epstein, Appl. Phys. Lett., 2005, 86, 154107.

26 B. C. Edwards, J. E. Anderson, R. I. Epstein, G. L. Mills and A. J. Mord, J. Appl. Phys., 1999, 86, 6489.

27 A. Rayner, M. E. J. Friese, A. G. Truscott, N. R. Heckenberg and H. Rubinsztein-Dunlop, J. Mod. Opt., 2001, 48, 103.

28 M. T. Murtagh, G. H. Sigel Jr, J. C. Fajardo, B. C. Edwards and R. I. Epstein, J. Non-Cryst. Solids, 1999, 253, 50.

29 A. Rayner, N. R. Heckenberg and H. Rubinsztein-Dunlop, J. Opt. Soc. Am. B, 2003, 20, 1037.

30 B. Heeg, M. D. Stone, A. Khizhnyak, G. Rumbles, G. Mills and P. A. DeBarber, Phys. Rev. A, 2004, 70, 021401.

31 D. V. Seletskiy, M. P. Hasselbeck and M. Sheik-Bahae, Appl. Phys. Lett., 2010, 96, 181106.

32 W. M. Patterson, D. V. Seletskiy, M. Sheik-Bahae, R. I. Epstein and M. P. Hehlen, J. Opt. Soc. Am. B, 2010, 27, 611.

33 J. R. Fernandez, Proc. SPIE, 2002, 4645, 135.

34 C. E. Mungan and T. R. Gosnell, Laser cooling of solids, in Advances in Atomic, Molecular, and Optical Physics, ed. B. Bederson and H. Walther, Academic, 1999, vol. 40, pp. 161-228.

35 J. Fernandez, A. Mendioroz, A. J. Garcia, R. Balda and J. L. Adam, Phys. Rev. B: Condens. Matter Mater. Phys., 2000, 62, 3213.

36 J. Fernandez, A. Mendioroz, A. J. Garcia, R. Balda, J. L. Adam and M. A. Arriandiaga, Opt. Mater., 2001, 16, 173.

37 S. R. Bowman and C. E. Mungan, Appl. Phys. B, 2000, 71, 807.

38 R. I. Epstein, J. J. Brown, B. C. Edwards and A. Gibbs, J. Appl. Phys., 2001, 90, 4815.

39 A. Mendioroz, J. Fernandez, M. Voda, M. Al-Saleh, R. Balda and A. J. Garcia-Adeva, Opt. Lett., 2002, 27, 1525.

40 S. Bigotta, D. Parisi, L. Bonelli, A. Toncelli, M. Tonelli and A. Di Lieto, J. Appl. Phys., 2006, 100, 013109.

41 S. Bigotta, D. Parisi, L. Bonelli, A. Toncelli, A. D. Lieto and M. Tonelli, Opt. Mater., 2006, 28, 1321.

42 S. Bigotta, A. Di Lieto, D. Parisi, A. Toncelli and M. Tonelli, Proc. SPIE, 2007, 6461, 64610E.

43 S. Bigotta, Nuovo Cimento B, 2007, 122, 685694. 
44 D. Seletskiy, M. P. Hasselbeck, M. Sheik-Bahae, R. I. Epstein, S. Bigotta and M. Tonelli, Proc. SPIE, 2008, 6907, 69070B.

45 J. V. Guiheen, C. D. Haines, G. H. Sigel, R. I. Epstein, J. Thiede and W. M. Patterson, Phys. Chem. Glasses: Eur. J. Glass Sci. Technol., Part B, 2006, 47, 167.

46 C. W. Hoyt, M. Sheik-Bahae, R. I. Epstein, B. C. Edwards and J. E. Anderson, Phys. Rev. Lett., 2000, 85, 3600.

47 C. W. Hoyt, M. P. Hasselbeck, M. Sheik-Bahae, R. I. Epstein, S. Greenfield, J. Thiede, J. Distel and J. Valencia, J. Opt. Soc. Am. B, 2003, 20, 1066.

48 W. Patterson, S. Bigotta, M. Sheik-Bahae, D. Parisi, M. Tonelli and R. Epstein, Opt. Express, 2008, 16, 1704.

49 J. Fernandez, A. J. Garcia-Adeva and R. Balda, Phys. Rev. Lett., 2006, 97, 033001.

50 N. J. Condon, S. R. Bowman, S. P. O'Connor, R. S. Quimby and C. E. Mungan, Opt. Express, 2009, 17, 5466.

51 M. P. Hehlen, R. I. Epstein and H. Inoue, Phys. Rev. B: Condens. Matter Mater. Phys., 2007, 75, 144302.

52 W. M. Patterson, P. C. Stark, T. M. Yoshida, M. Sheik-Bahae and M. P. Hehlen, J. Am. Ceram. Soc., 2011, 94, 2896-2901.

53 S. D. Melgaard, D. V. Seletskiy, A. Di Lieto, M. Tonelli and M. Sheik-Bahae, Opt. Lett., 2013, 38, 1588-1590.
54 D. V. Seletskiy, S. D. Melgaard, R. I. Epstein, A. Di Lieto, M. Tonelli and M. Sheik-Bahae, J. Lumin., 2013, 133, 5-9.

55 D. V. Seletskiy, S. D. Melgaard, R. I. Epstein, A. Di Lieto, M. Tonelli and M. Sheik-Bahae, Opt. Express, 2011, 19, 18229-18236.

56 D. Herriott, H. Kogelnik and R. Kompfner, Appl. Opt., 1964, 3, 523-526.

57 S. D. Melgaard, Cryogenic Optical Refrigeration: Laser cooling of solids below 123 K, Ph.D. dissertation, University of New Mexico, Albuquerque, NM, 2013.

58 D. V. Seletskiy, S. D. Melgaard, A. Di Lieto, M. Tonelli and M. Sheik-Bahae, Opt. Express, 2010, 18, 18061-18066.

59 R. Judkoff and F. Sokol, Performance of a selective-surface Trombe wall in a small commercial building, Solar Energy Research Institute Report \#TP-721-1158, Boulder, CO, 1981.

60 J. J. Mason and T. A. Brendel, Proc. SPIE, 1982, 324, 139-145. 61 R. I. Epstein and M. Sheik-Bahae, Optical Refrigeration in Solids: Fundamentals and Overview, in Optical Refrigeration: Science and Applications of Laser Cooling of Solids, ed. R. I. Epstein and M. Sheik-Bahae, Wiley-VCH Verlag, Weinheim, 2009. 